\title{
Effects of Different Cyclic Pressurization and Relief Patterns on Heel Skin Blood Perfusion
}

Harvey N. Mayrovitz, PhD, and Nancy Sims, RN

$\mathbf{P}$ ressure ulcers due to sustained unrelieved or inadequately relieved pressure is an important clinical, humanitarian, and economic problem. ${ }^{1-3}$ Although many factors are involved, ${ }^{4-6}$ a final common pathway is associated with blood flow changes within pressure-loaded tissue. These detrimental blood flow changes affect skin breakdown in multiple ways, ${ }^{7-8}$ with the greatest frequency at sites of bony prominences. The heel is particularly prone to such effects, ${ }^{9}$ in part because of its relatively lower resting blood perfusion level, ${ }^{10}$ higher amounts of surface pressure when under load,, $11-14$ and the possibility of compromised local blood flow if lower-extremity arterial disease is present. ${ }^{10}$

Skin breakdown processes are affected by decreases in local blood flow during heel loading ${ }^{10}$ and by flow recovery features after unloading. ${ }^{15-17}$ Practical strategies that would efficiently minimize the detrimental effects of pressure have remained elusive. Various approaches, often based on interface pressure characteristics of devices and support surfaces, ${ }^{18-21}$ have had less than optimum results. A basis for a potentially effective management strategy has evolved from previous work. ${ }^{10-11,15-17}$ The most directly relevant finding ${ }^{17}$ was that when heels are pressure loaded for different durations with the same pressure or with different pressures for the same duration, a load- and duration-dependent blood flow hyperemia results when pressure is relieved.

It was hypothesized that if a practical balance between pressurization and pres-

\section{ABSTRACT}

OBJECTIVE: It was hypothesized that a device or support surface providing intermittent cycles of pressurization and pressure relief might minimize the impact of blood flow deficits in the heels resulting from the application of pressure. Because this possibility depends on whether pressure-relief hyperemia can adequately compensate for blood flow deficits, the main objective was to determine how different temporal patterns of pressurization and pressure relief would affect average skin blood perfusion of the heels.

DESIGN: Using a laser Doppler, skin blood perfusion was measured in the heels of 20 healthy subjects while they lay supine for $\mathbf{8 0}$ minutes on a support surface. The end cell supporting the heel produced 3 different cyclic patterns of pressurization and either full or partial pressure relief. Each pattern of 1, 2, or 4 cycles was contained within contiguous 20-minute intervals. Skin blood perfusion was determined during full pressurization and during pressure relief for $\mathbf{2}$ protocols with 10 subjects each.

SETTING: University research center

MAIN OUTCOME MEASURE: Overall average skin blood perfusion in relation to baseline

RESULTS: Full pressure relief yielded a significantly greater skin blood perfusion than partial relief. However, whether pressure relief was full or partial, the average skin blood perfusion of each cyclic pattern was greater than baseline.

CONCLUSION: Consistent with the proposed hypothesis, pressure-relief cycles resulted in an average heel skin blood perfusion that was greater than resting baseline. In the healthy subjects studied, this occurred because hyperemia during pressure relief compensated for flow deficits during pressurization. These results are applicable when the patient is capable of a normal physiologic hyperemic response. The next major investigative challenge is to examine the impact of pressure-relief cycles on patients with diminished hyperemic reserve.

ADV SKIN WOUND CARE 2002;15:158-160, 162-4.

sure relief provided by a device or support surface could be found, then the impact of flow deficits during the application of pressure might be minimized. This strategy intrinsically depends on the ability of pressure-relief hyperemia to adequately compensate for intervals of flow deficits, rendering average heel flow near normal.
It is unknown whether different temporal patterns of heel pressure and relief would have a greater effect on average blood flow than full pressure-relief intervals. To

Harvey N. Mayrovitz, PhD, is Professor of Physiology and Nancy Sims, RN, is Research Associate at the College of Medical Sciences, Nova Southeastern University, Ft Lauderdale, FL. Submitted May 21, 2001; accepted in revised form September 26, 2001. 
Figure 1. POSITION OF THE HEEL ON THE END CELL OF THE SUPPORT SURFACE

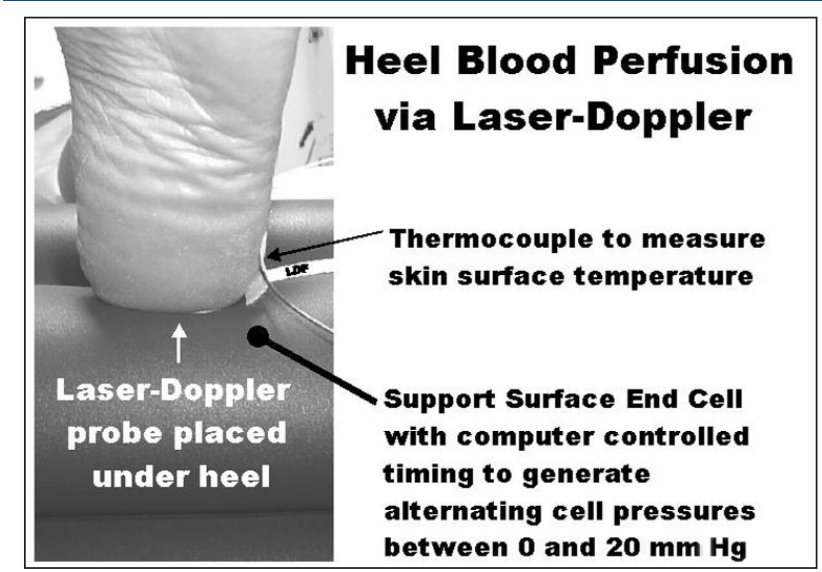

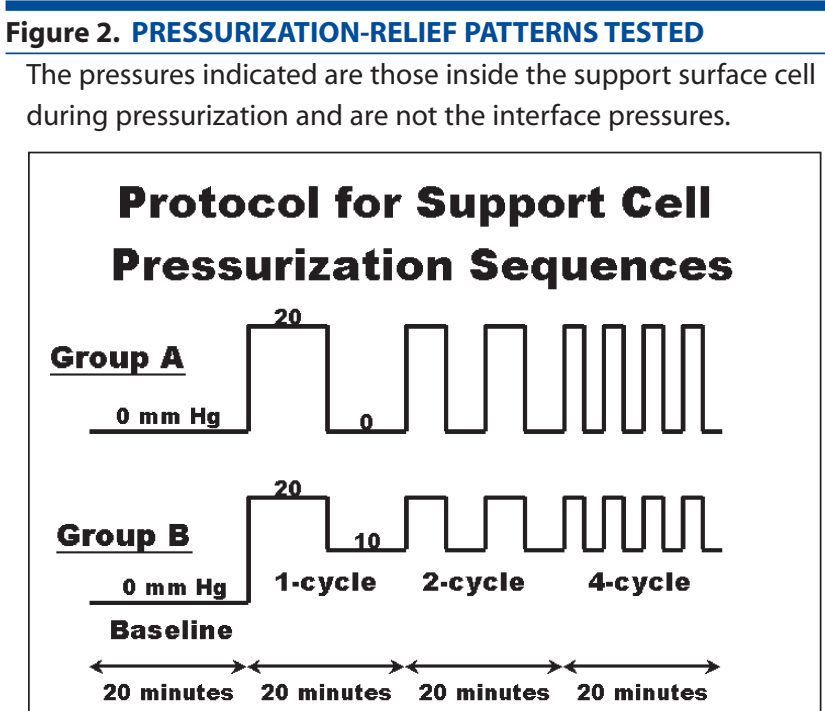

investigate this, skin blood perfusion (SBF) was measured in the heels of 20 healthy subjects while they lay supine for 80 minutes on a support surface capable of producing different temporal patterns of heel pressure and pressure relief. The primary goal was to characterize the effects of dynamic heel support patterns that differed with respect to pressure and pressure-relief intervals.

\section{METHODS}

\section{Subjects}

Twenty volunteers from the medical school student and staff population were tested after signing an approved institutional review board consent form. All subjects were free of lower-extremity vascular disease, which was verified by pretest ankle-brachial pressure indices (determined by a blood pressure cuff and Doppler ultrasound). None were taking medications that would impact vascular reactivity. There were 5 men and 5 women in each group. Subjects were randomly divided into either group A or B; each group followed 1 of 2 support pattern protocols. For groups A and B, respectively, there were no significant differences in age (29.8 \pm 1.7 vs $31.3 \pm 3.5$ years), height
(66.7 \pm 0.8 vs $67.0 \pm 1.1$ inches), or weight (140 \pm 30 vs $140 \pm 26$ pounds).

\section{Protocol and support patterns}

Subjects lay on a support surface with their left heel positioned on the end support cell. Pressure in the end support cell was computer controlled and could be adjusted to vary between an upper and lower limit on a cyclic basis by increasing or decreasing the air volume in the cell. Cell pressure was sensed by a pressure transducer in the cell with a feedback signal to an actuator valve that controlled the airflow to or from the cell (Figure 1). The dynamic patterns consisted of 3 distinct, sequential, 20-minute intervals in which either 1, 2, or 4 full cycles were applied for 60 minutes (Figure 2). For group A ( $\mathrm{n}=$ 10), the internal cell pressure supporting the heel cycled between $20 \mathrm{~mm} \mathrm{Hg}$ and 0 mm Hg. For group B ( $n=10)$, the cell pressure supporting the heel cycled between $20 \mathrm{~mm} \mathrm{Hg}$ and $10 \mathrm{~mm} \mathrm{Hg}$. The half cycle length for 1, 2, and 4 cycles was 10, 5, and 2.5 minutes, respectively. For each group, the sequential order of the dynamic pattern was 4-2-1 in half the subjects and 1-2-4 in the other half. The first cyclic pattern was initiated after a baseline interval of 20 minutes in which the heel was not loaded ( $0 \mathrm{~mm} \mathrm{Hg}$ ). There were no significant differences in room temperature during the tests $(23.4 \pm$ $0.06^{\circ} \mathrm{C}$ for group $\mathrm{A}$ vs $23.7 \pm 0.07^{\circ} \mathrm{C}$ for group $\mathrm{B})$.

\section{Blood perfusion}

Heel SBF was monitored with a laser Doppler probe affixed to the heel with tape and connected to a perfusion monitor (Vasamedics model 403a; Vasamedics, St Paul, MN). The position of the probe was at the site of heel contact with the support surface (Figure 1). There was no callous on the heel at the site of contact with the support surface where the Doppler probe was placed. The probe, which was $10 \mathrm{~mm}$ wide and $1 \mathrm{~mm}$ high, was placed on the posterior protuberance such that contact was flat against the support surface. Skin blood perfusion was continuously monitored throughout the experimental sequence. Skin temperature was measured with a thermocouple near the site of SBF measurement. Average skin temperature for both groups combined was $27.5 \pm 0.5^{\circ} \mathrm{C}$, with no significant temporal changes detected.

\section{Interface pressure}

At the end of the 80-minute sequence, heel interface pressures were measured 
Figure 3. EXAMPLE OF BLOOD PERFUSION RESPONSES TO PRESSURIZATION AND RELIEF

A full protocol sequence is shown in which the cell pressure cycles from 0 to $20 \mathrm{~mm} \mathrm{Hg}$ for 1-, 2-, and 4-cycle patterns.

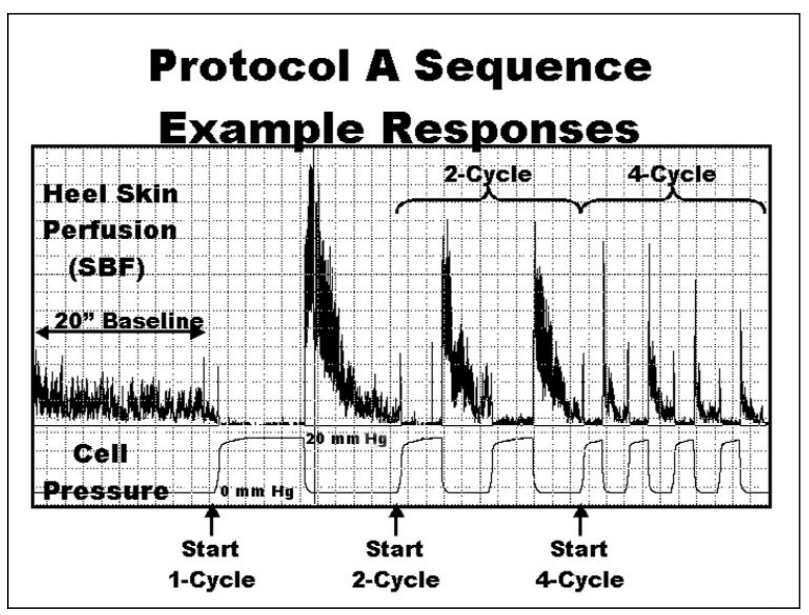

Figure 4. COMPARISON OF FULL VERSUS PARTIAL PRESSURE RELIEF

Complete cell venting to a cell pressure of $0 \mathrm{~mm} \mathrm{Hg}$ resulted in a significantly greater hyperemia than did partial venting.

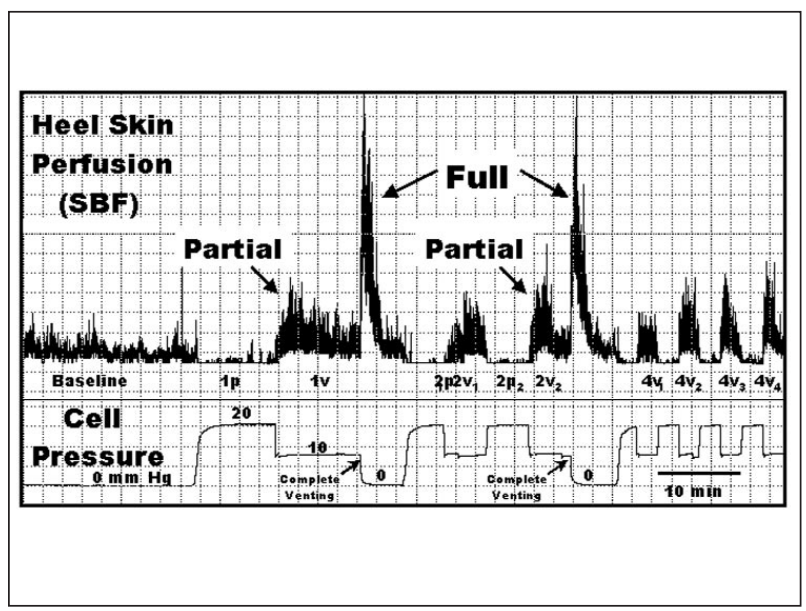

with a pressure sensor that was placed between the heel and the supporting cell. A Banda Press pressure sensor (Model BP02; Bioscience Research Institute, Ft Lauderdale, FL) was placed between the heel and supporting cell. The cells were pressurized to the levels corresponding with those used during the test sequence. Six measurements of interface pressure were taken for each subject at a cell pressure of $20 \mathrm{~mm} \mathrm{Hg}$, and 6 additional measurements were taken with the cell pressurized to $10 \mathrm{~mm} \mathrm{Hg}$ for group B subjects. Averages of the 6 measurements were used to report interface pressures.

\section{Assessment parameters and data analysis}

The main comparison parameter was the average SBF during each 20-minute interval in relation to the baseline average SBF obtained at zero pressure by computer integration. For cyclic support intervals, average SBF was determined for each cycle by summing the average SBF during maximum and minimum pressure phases. For 2- and 4-cycle patterns, the average SBF in each cycle was used to characterize the pattern's overall average. For each subject, SBF in each cycle interval was compared with baseline $\mathrm{SBF}$ using the ratio $\mathrm{SBF}_{\mathrm{r}}=$ $\left(\mathrm{SBF}_{\mathrm{j}} / \mathrm{SBF}_{\text {base }}\right)$, in which $\mathrm{j}=1-3$ and corresponded to the 1-, 2-, and 4-cycle pattern intervals. Statistical comparisons between groups used nonparametric Mann-Whitney tests, with an alpha level less than 0.05 considered statistically significant.

\section{RESULTS}

\section{Interface pressure}

With the internal pressure of the end support cell set at its maximum of $20 \mathrm{~mm} \mathrm{Hg}$, heel interface pressures ranged from 55 to $147 \mathrm{~mm} \mathrm{Hg}$ (mean = $92 \pm 5$ mm Hg, $\mathrm{N}=$ 20). The interface pressures recorded for group B subjects $(n=10)$ was $81.8 \pm 4.6$ $\mathrm{mm} \mathrm{Hg}$, which was significantly less than that recorded for group A subjects (mean = $101.2 \pm 7.4 \mathrm{~mm} \mathrm{Hg}, P=.016)$. With the cell pressure set at $10 \mathrm{~mm} \mathrm{Hg}$ (group B subjects only), interface pressures ranged from 35 to $74 \mathrm{~mm} \mathrm{Hg}$ (mean $=48 \pm 4 \mathrm{~mm} \mathrm{Hg}$, $\mathrm{n}=10$ ), which was approximately half of what was achieved with a cell pressure of $20 \mathrm{~mm} \mathrm{Hg}$. For group A, there was a sig- nificant $(P<.01)$ correlation between interface pressure and subject height $(R=$ $0.775)$ and weight $(R=0.765)$. For group $B$, there was a similar tendency for interface pressure to increase with height; however, the correlation was not statistically significant. There was no detectible overall correlation between interface pressure and SBF response for any support cycles or patterns.

\section{Skin blood perfusion responses}

Cell pressurization was associated with a decrease in SBF; pressure relief was associated with a hyperemic response (Figure 3). The magnitude of the increase in SBF depended on whether the release was full (to $0 \mathrm{~mm} \mathrm{Hg}$ ) or partial (to $10 \mathrm{~mm}$ $\mathrm{Hg}$ ) (Figure 4)

\section{Skin blood perfusion during full pressurization}

Pressurizing the end support cell to 20 $\mathrm{mm} \mathrm{Hg}$ resulted in a decrease in heel SBF to levels that ranged from $0.12 \pm 0.05$ to $0.44 \pm 0.13$ of baseline (Figure 5). Although subjects in groups $\mathrm{A}$ and $\mathrm{B}$ were exposed to the same amount of maximum cell pressure, the mean 


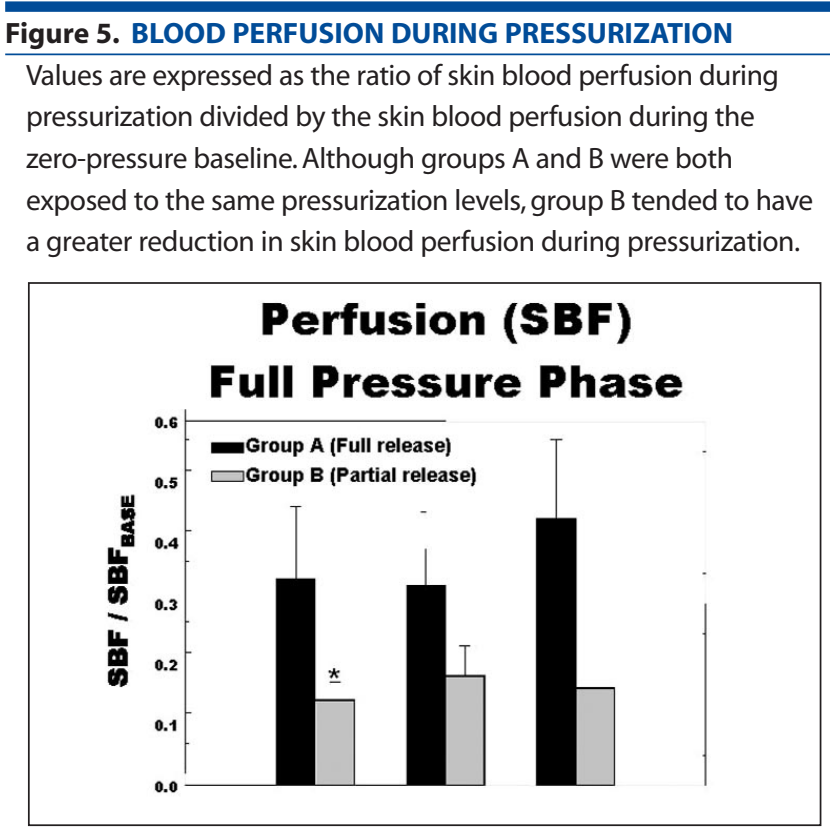

Bars are standard error of the mean $* P<.05$ between groups $A$ and $B$

Figure 7. AVERAGE PERFUSION OVER FULL PRESSURIZATION AND PRESSURE-RELIEF CYCLES

Values are expressed as the ratio of average skin blood perfusion during each pressure-relief cycle divided by the skin blood perfusion during the zero-pressure baseline. Full relief patterns were associated with a significantly greater average perfusion than partial relief patterns.

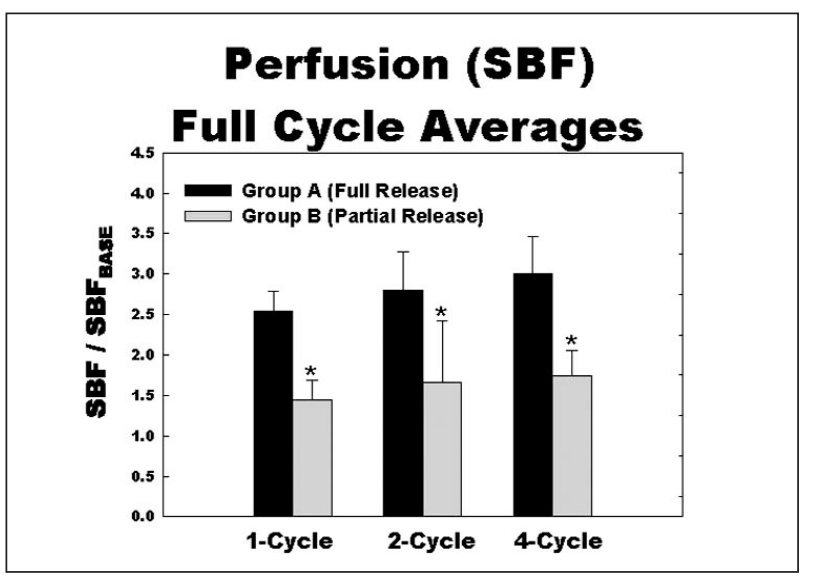

${ }^{*} P<.01$ for group $A$ vs group $B$

decrease in SBF relative to baseline was, on average, larger in group B; however, only for the 1-cycle pattern that had 10 minutes of continuous loading was the difference

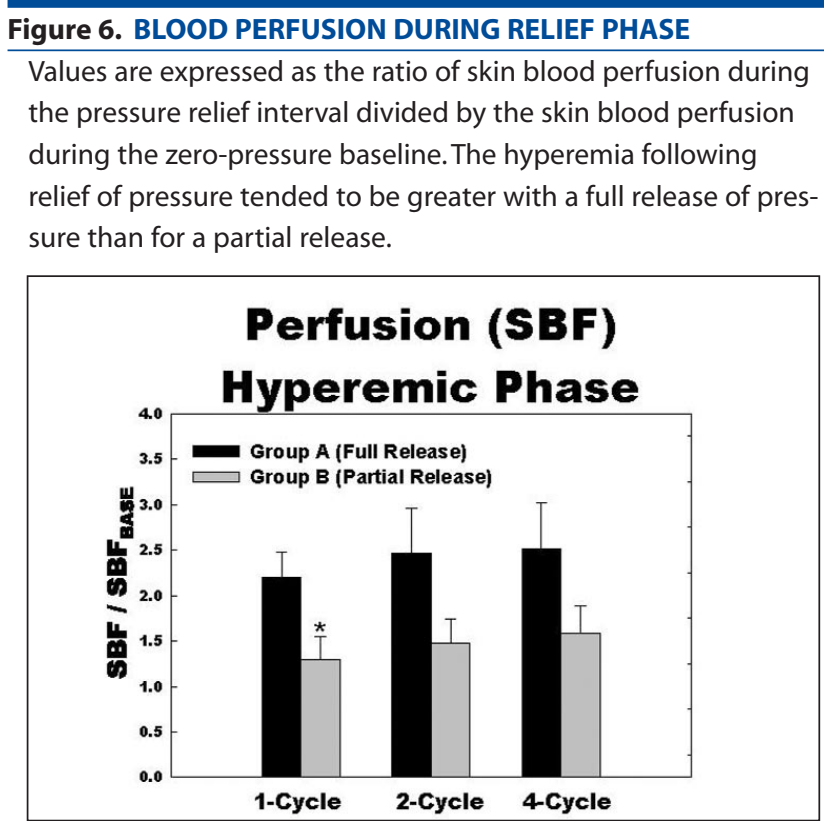

statistically significant $(P=.028)$. The greater $\mathrm{SBF}$ reduction in group B is not readily explained on the basis of interface pressure values because these values were less for group B than for group $\mathrm{A}$.

\section{Skin blood perfusion during pressure release}

Release of the cell pressure resulted in a hyperemic response (Figure 6); the magnitude of the response depended on whether the release was full or partial. During a full release interval, SBF achieved a value that, on average, was at least twice that of the average SBF during baseline. Partial release of pressure was associated with a lesser increase. For the 1- cycle pattern, the relative hyperemia was significantly less for partial releases (1.3 \pm $0.25 \mathrm{~mm} \mathrm{Hg})$ than for full releases $(2.2 \pm 0.28$ $\mathrm{mm} \mathrm{Hg}, P=.03)$. For the 2 - and 4 -cycle patterns, differences between full and partial release responses were similar, but the difference was not statistically significant.

\section{Skin blood perfusion during full cycles}

Skin blood perfusion, averaged over each test cycle interval of pressurization and relief, was the main parameter of interest with respect to the effects of heel loading pattern. Results show that the average full-cycle SBF was significantly greater $(P<.01)$ than the skin blood perfusion averaged over the baseline interval. This was true for all cycle patterns of each protocol. Analysis of variance showed an overall statistically significant difference between groups, with the partial release having a lower full-cycle average skin blood perfusion (Figure 7). Comparisons of individual cyclic patterns between groups revealed $P$ values for $1-, 2-$, and $4-$ cycle patterns of .005, .049, and .042, 
respectively. Using analysis of variance for repeated measures, examination of within group effects revealed no significant difference among cycle patterns for either group A or B.

\section{DISCUSSION}

\section{Assessment approach}

The main findings of this study relate to the effects of dynamic heel support patterns that differed with respect to support pressure relief frequency and the magnitude of the relief pressure level. Impacts of the different cyclic patterns were assessed via the relative perfusion in the different 20-minute cyclic intervals in relation to baseline. The rationale of this approach is that overall clinical utility of one pattern versus another depends on overall perfusion effects, which intrinsically depend on both pressurization and relief cycles. Because each subject's baseline perfusion is individual to that subject, effects of cycle pattern on net blood perfusion are suitably, if not best, assessed via the chosen ratios.

\section{Main findings}

The most substantially supported result of the present study indicates that complete heel off-loading during pressurerelief phases yields greater relative perfusion when compared with partial offloading. The difference is likely due to the fact that partial off-loading in the amount used in the present investigation blunts the normal hyperemic response magnitude. Because hyperemia magnitude during pressure relief contributes most to average perfusion, its blunting would account for the findings. In both protocols, however, average perfusions associated with each cycle pattern were greater than baseline because the pressure-relief hyperemia more than compensated for the flow deficits during the heel loading phases. This result is consistent with the hypothesis that a relative normalization of average heel skin perfusion may be possible with properly chosen pressurerelief patterns.

The second finding suggests that, of the cycle patterns studied, the 4-cycle pattern is most consistent with minimal detrimental effects, judged by the resulting full-cycle average SBF. In both groups, the 4-cycle pattern was associated with the highest mean perfusion ratio when compared with baseline. There was also a slight trend for this ratio to rise from 1-cycle through 4-cycle patterns, although the trend was not statistically significant in either group.

\section{Interface pressure}

The absence of any relationship between interface pressure and SBF decrements or hyperemia suggests that interface pressure by itself is an inadequate parameter to assess the impact of pressure loading on heel tissue and vascular structures. This is in accord with the concept that heel tissue structure, geometry, and properties are primary determinants of interface pressure transmission and distribution to underlying vascular structures for any given person and loading situation. This indicates that SBF is the better direct parametric measure of heel support effects and suggests that its assessment may identify new risk factors for the likelihood of heel breakdown under prescribed conditions of loading.

\section{SUMMARY}

This initial investigation of the effects of various cyclical pressure patterns for supporting the heel has demonstrated clear differences between full and partial pressure-relief approaches. Full relief is associated with an average heel blood perfusion greater than that during resting baseline. This phenomenon occurs because the hyperemia during the relief phase more than compensates for the flow deficit during heel loading. Partial relief blunts this normal response, causing less hyperemia, but still results in an average perfusion that exceeds baseline. No specific cycle length tested showed a significant advantage with respect to achieving a higher average perfusion. The slight upward trend in relative perfusion from 1through 4-cycle patterns suggests a benefit for the 4-cycle approach, but this is not supported by adequate statistical evidence. In addition, these results are strictly applicable when a person has the capability of a normal physiologic hyperemic response. It is unknown what impact depressed vascular responsiveness and/or diminished hyperemic reserve would have on the basic blood perfusion findings. This issue represents the next major investigative challenge.

\section{REFERENCES}

1. Allman R. Pressure ulcer prevalence, incidence, risk factors, and impact. Clin Geriatr Med 1997;13:421-36.

2. Barczak CA, Barnett RI, Childs EJ, Bosley LM. Fourth National Pressure Ulcer Prevalence Survey. Adv Wound Care 1997;10(4):18-26.

3. Schue RM, Langemo DK. Pressure ulcer prevalence and incidence and a modification of the Braden Scale for a rehabilitation unit. J Wound Ostomy Continence Nurs 1998;25(1):36-43.

4. Kemp M, Keithley J, Smith D, Morreale B. Factors that contribute to pressure sores in surgical patients. Res Nurs Health 1990;10:293-301.

5. Tourtual DM, Riesenberg LA, Korutz CJ, et al. Predictors of hospital acquired heel pressure ulcers. Ostomy Wound Manage 1997;43(9):2434.

6. Byers P, Carta C, Mayrovitz HN. Pressure ulcer considerations in surgical patients. Adv Skin Wound Care 2000;13:115-21.

7. Kosiak M. Etiology and pathology of ischemic ulcers. Arch Phys Med Rehabil 1959;40:62-9.

8. Kosiak M. Etiology of decubitus ulcers. Arch Phys Med Rehabil 1961;42:19-29.

9. Graff MK, Bryant J, Beinlich N. Preventing heel breakdown. Orthop Nurs 2000;19(5):63-9.

10. Abu-Own A, Sommerville K, Scurr JH, ColeridgeSmith PD. Effects of compression and type of bed surface on the microcirculation of the heel. Eur J Vasc Endovasc Surg 1995;9:327-34.

11. Ek A, Gustavsson G, Lewis D. Skin blood flow in relation to external pressure and temperature in the supine position on a standard hospital mattress. Scand J Rehabil Med 1987;19:121-6. 
12. Counsell C, Seymour S, Guin P, Hudson A. Interface skin pressures on four pressure-relieving devices. J Enterostomal Ther 1990;17:150-3.

13. Allen V, Ryan DW, Murray A. Potential for bed sores due to high pressures: influence of body sites, body position, and mattress design. $\mathrm{Br} J$ Clin Pract 1993;47:195-7.

14. Allen V, Ryan DW, Murray A. Measurements of interface pressure between body sites and the surfaces of four specialised air mattresses. $\mathrm{Br} \mathrm{J}$ Clin Pract 1994;48:125-9.

15. Mayrovitz HN, Smith J, Delgado M, Regan MB. Heel blood perfusion responses to pressure loading and unloading in women. Ostomy Wound Manage 1997;43(7):16-26.

16. Mayrovitz HN, Smith J. Heel-skin microvascular blood perfusion responses to sustained pressure loading and unloading. Microcirculation 1998;5:227-33.

17. Mayrovitz HN, Smith J. Blood perfusion hyperemia in response to graded loading of human heels assessed by laser-Doppler imaging. Clin Physiol 1999;19:351-9.

18. Guin P, Hudson A, Gallo J.The efficacy of six heel pressure reducing devices. Decubitus 1991; 4(3):15-20.

19. Hover AE, Krouskop TA. Pressure relief characteristics of a new foam overlay: a preliminary performance evaluation. J ET Nurs 1992; 19(2):42-7.

20. De Keyser G, Dejaeger E, De Meyst H, Eders GC. Pressure-reducing effects of heel protectors. Adv Wound Care 1994;7(4):30-4.

21. Ryan DW, Allen V, Murray, A. An investigation of interface pressures in low air loss beds. Int J Clin Pract 1997;51:296-8.

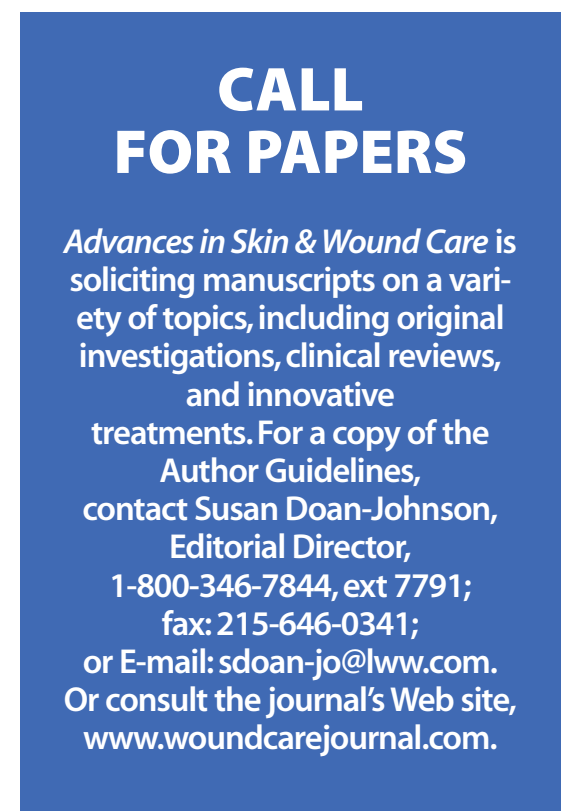

